\title{
When is a biomineral not a biomineral? Multi-scale complexity of iron oxide crystallisation in the Gunflint Chert
}

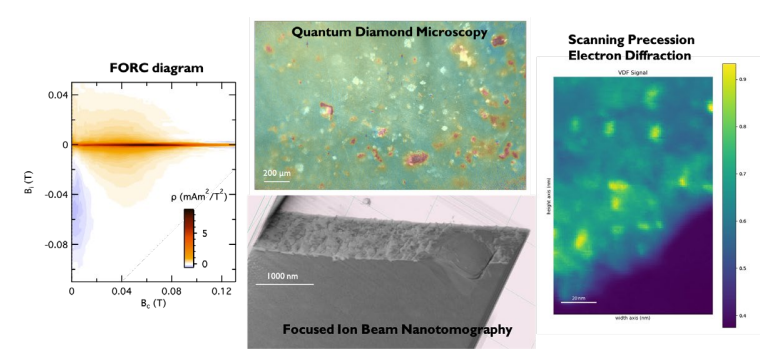

\author{
JOSHUA FRANZ EINSLE ${ }^{1}$, IOAN LASCU ${ }^{2}$, ANASTAS \\ POPRATILOFF ${ }^{3}$, ROGER FU $^{4}$ AND IAN MACLAREN ${ }^{1}$ \\ ${ }^{1}$ University of Glasgow \\ ${ }^{2}$ Smithsonian Museum of Natural History \\ ${ }^{3}$ The George Washington University \\ ${ }^{4}$ Harvard University \\ Presenting Author: joshuafranz.einsle@glasgow.ac.uk
}

Previous studies have reported putative magnetofossils from Precambrian rocks in a variety of localities around the globe. Identifying and studying magnetic particles within these rocks would allow a better understanding of iron biomineralization, the origin of magnetotaxis, the length of the Earth's fossil record, and the history of the geodynamo. Metagenomic evidence for some magnetotactic bacteria lineages reaching as far back as the Archean, coupled with findings of magnetic particles akin to magnetofossils from Precambrian rocks, provided the impetus for the present multi-scale magnetic and electron microscopy analysis of chert samples from the $1.9 \mathrm{Ga}$ Gunflint Formation. We use a correlative approach that bridges bulk rock magnetic measurements with high spatial resolution magnetic and structural characterisation. First-order reversal curves (FORCs) and low-temperature magnetometry indicate that the magnetic carrier in the Gunflint chert has the fingerprint of single domain, non-interacting magnetite nanoparticles. Quantum diamond microscopy shows that the sources of magnetisation are at the interface between the chert matrix and

Fe-Mn carbonates. Focused ion beam (FIB) nanotomography at this interface reveals a thin layer $(50-100 \mathrm{~nm})$ of iron oxide with an irregular tabular morphology. We investigate the complex nanocrystalline structure of this interface using scanning electron diffraction. We recorded diffraction patterns every $1 \mathrm{~nm}$ and interrogated the diffraction space using multivariate statistics. From this approach we extracted a virtual dark field image of the 111 iron oxide diffraction ring. We found the interface to be composed of superparamagnetic magnetite particles $(10 \mathrm{~nm})$ arranged in clusters. Strong magnetostatic interactions between these particles result in uniaxial switching when a magnetic field is applied, explaining the observed bulk single domain-like magnetic behavior. The magnetite interface formed through the thermal decomposition of the Fe-Mn carbonates in the presence of fluids during a low-grade metamorphic event, likely associated with the Grenville Orogeny. This study underscores the

importance of microanalysis in understanding bulk rock and mineral properties. Through targeted use of multi-scale microscopy we show that the bulk magnetic signature is not result of the presence of magnetofossils, but rather an emergent property resulting from the interaction of smaller magnetite grains arranged in clusters. 